\title{
Agricity- An Agricultural Information System
}

\author{
M.Sunadarababu*, S.Sai Kumar*, Dr.B.V.Subba Rao*, Dr. J.Rajendra Prasad*, \\ L.Ravi Kumar*, K.Swaroopa Rani* \\ *Department of Information Technology, PVP Siddhartha Institute of Technology, Andhra Pradesh, India \\ Email:sundar539@gmail.com,saikumar.senagavarapu@gmail.com
}

\begin{abstract}
Agricity is a agricultural information system that provides its users and researches to get online information about, the crop, statistical details and provide products based on this information. The trends of the crops act so that these will be pretty important to the users who access these via the Internet. The main features of the information system includes information retrieval facilities for users from anywhere in the form of obtaining statistical information about fertilizer, research institutes and researches, suitable soil concentration for the corresponding crops, statistical information about exports and etc. In addition this provides individual information about products which include selling products. The system allows the retrieving facilities but also the updating facilities to the authorized persons in the corresponding institutes. HTML, CSS, JS are used to create the front end for the system and SQL Server was used for the back end. The graphical user interface of the front end use Java Applets, ASP Interfaces and access the back end SQL Server Database using embedded SQL Queries for the retrieval and update. The front end and back-end is connected using a SQL Server. They will be able to use this via Internet. Users will be given login name and password so that they can log-in to the database.
\end{abstract}

Keywords-Agricity, MySQL, SQL Server.

\section{INTRODUCTION}

Agricity is a agricultural information system that provides its users and researches to get online information about, the crop, statistical details and provide products. E-agriculture is a rising field for enhancing existing agriculture and food security through enhanced processes for knowledge access and it acts as a switch using information and communication technologies. In short e-Agriculture will connect all concerned persons starting from farmers to researchers together. Farmers can get the desired information at any instant of time from any part of world and they can also get the help from experts viewing their problem immediately by without moving anywhere. The trends of the crops act so that these will be pretty important to the users who access these via the Internet. The main features of the information system includes information retrieval facilities for users from anywhere in the form of obtaining statistical information about fertilizer, research institutes and researches, suitable soil concentration for the corresponding crops, statistical information about exports and etc. In addition this provides individual information about products which include selling products. The system allows the retrieving facilities but also the updating facilities to the authorized persons in the corresponding institutes.

Previously, we have many websites and applications regarding Agriculture which provide certain information about agriculture products like seeds, fertilizers, cereals and many other but they are not providing entire sufficient details about the products and the representing model is tough and complicated. Many websites are present from olden days but no one is interested in that as they are difficult to use.

- There are many websites available in olden days but they don't have efficient information.

- The payment option is very difficult and not easy to understand in other websites.

\section{PROPOSED WORK}

For improving agricultural productivity an expert agricultural advice is given to the farmers both in a timely and personalized situation. Based on this advice, the farmers can buy products here itself at reasonable costs. Here, in this system agricultural experts generate the advice by using the modern agriculture which is highly knowledge intensive which also requires timely, reliable and accurate information on natural resource endowments and their usage patterns at present and future technology available for their utilization and other information about markets, weather, subsidy, etc.

The news releases from the government does not reach the farmers in time, therefore an alert system is being built for daily releases and for seasonal releases.HTML, CSS, JS are used to create the front end for the system and SQL Server was 
used for the back end. The front end and back-end is connected using a SQL Server.

They will be able to use this via Internet. Users will be given login name and password so that they can log-in to the database.

\section{SYSTEM IMPLEMENTATION Introduction to PhP:}

PHP is a server-side scripting language designed for web development but also used as a general-purpose programming language. As of January 2013, PHP was installed on more than 240 million websites (39\% of those sampled) and 2.1 million web servers. Originally created by Rasmus Lerdorf in 1995, the reference implementation of PHP is now produced by The PHP Group. While PHP originally stood for Personal Home Page, it now stands for PHP: Hypertext Preprocessor, a recursive backronym.

PHP code is interpreted by a web server with a PHP processor module, which generates the resulting web page: PHP commands can be embedded directly into an HTML source document rather than calling an external file to process data. It has also evolved to include a command-line interface capability and can be used in standalone graphical applications.

PHP is free software released under the PHP License. PHP can be deployed on most web servers and also as a standalone shell on almost every operating system and platform, free of charge.

\section{Database Connectivity}

Before we can access data in a database, we must open a connection to the MySQL server. In PHP, this is done with the mysqli_connect() function. Syntax:-

mysqli_connect(host,username,password,dbname);

Introduction to MySQL: MySQL is a fast, easy-touse RDBMS used for databases on many web sites. Speed was the developer's main focus from the beginning. In the interest of speed, they made the decision to offer fewer features than their major competitors (for instance, Oracle and Sybase). However, even though MySQL is less full featured than its commercial competitors, it has all the features needed by the large majority of database developers. It's easier to install and use than its commercial competitors.

MySQL is developed, marketed, and supported by MySQL AB, which is a Swedish company. The company licenses it in two ways:

- Open source software: MySQL is available via the GNU GPL (General Public License) for no charge. Anyone who can meet the requirements of the GPL can use the software for free.

- Commercial license: MySQL is available with a commercial license for those who prefer it to the GPL. If a developer wants to use MySQL as part of a new software product and wants to sell the new product, rather than release it under the GPL, the developer needs to purchase a commercial license.

\section{Advantages of Mysql:}

MySQL is a popular database with Web developers. Its speed and small size make it ideal for a Web site. It is open source, which means free, and we have the foundation of its popularity.

- It's fast. The main goal of the folks who developed MySQL was speed. Consequently, the software was designed from the beginning with speed in mind.

- It's inexpensive. MySQL is free under the open source GPL license, and the fee for a commercial license is very reasonable.

- It's easy to use. We can build and interact with a MySQL database by using a few simple statements in the SQL language, which is the standard language for communicating with RDBMSs.

- It can run on many operating systems. MySQL runs on a wide variety of operating systems - Windows, Linux, Mac OS, most varieties of UNIX (including Solaris, AIX, and DEC UNIX), FreeBSD, OS/2, Irix, and others.

- Technical support is widely available. A large base of users provides free support via mailing lists. The MySQL developers also participate in the e-mail lists. We can also purchase technical support from MySQL AB for a very small fee.

- It's secure. MySQL flexible system of authorization allows some or all database privileges (for example, the privilege to create a database or delete data) to specific users or groups of users. Passwords are encrypted.

\section{Introduction to Android:}

Android is a Linux-based operating system for mobile devices such as smartphones and tablet computers. It is developed by the Open Handset Alliance led by Google. Android has a large community of developers writing applications ("apps") that extend the functionality of the devices. Developers write primarily in a customized version of Java.

\section{Android Architecture:}

Android consists of a kernel based on the Linux kernel, with middleware, libraries and APIs written in $\mathrm{C}$ and application software running on an application framework which includes Javacompatible libraries based on Apache Harmony. Android uses the Dalvik virtual machine with justin-time compilation to run Dalvik dex-code (Dalvik 

ISSN : 2248-9622, Vol. 7, Issue 6, (Part-3) June 2017, pp.62-65

Executable), which is usually translated from Java bytecode.

The main hardware platform for Android is the ARM architecture. There is support for $\mathrm{x} 86$ from the Android x86 project, and Google TV uses a special $\mathrm{x} 86$ version of Android.
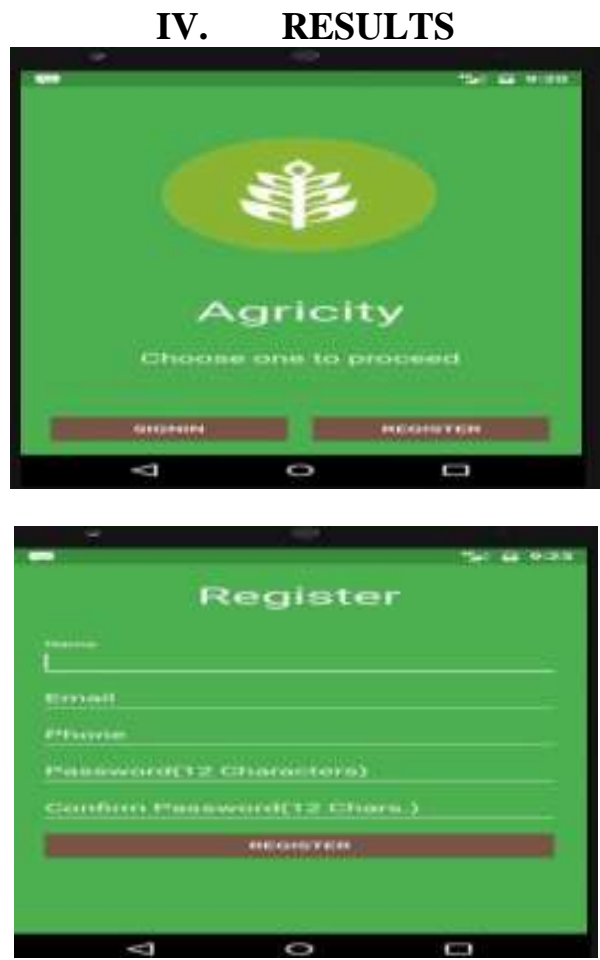

Fig 1: Home Page \& Registration Page
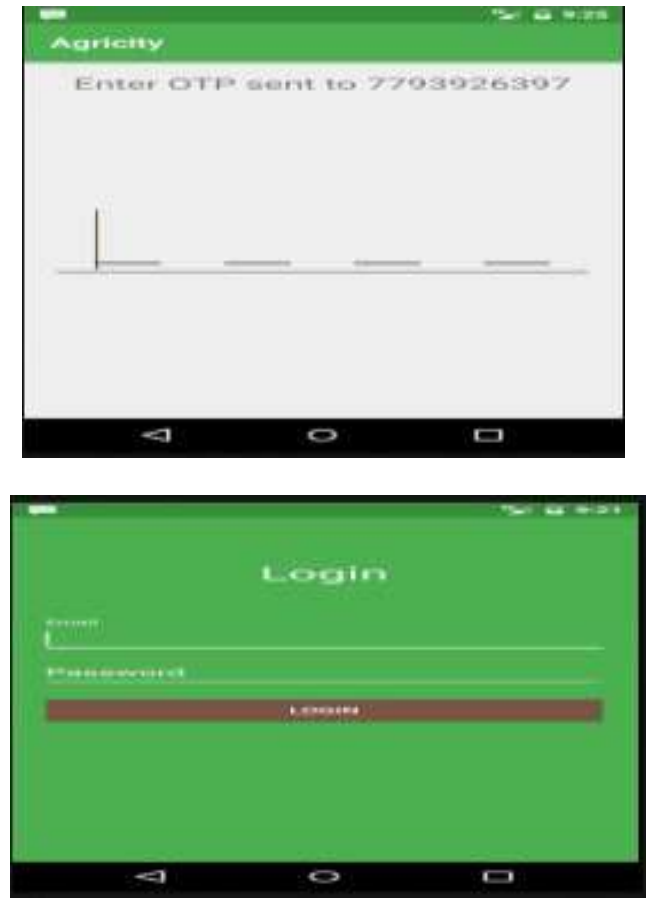

Fig 2: OTP Generation \& Login Page

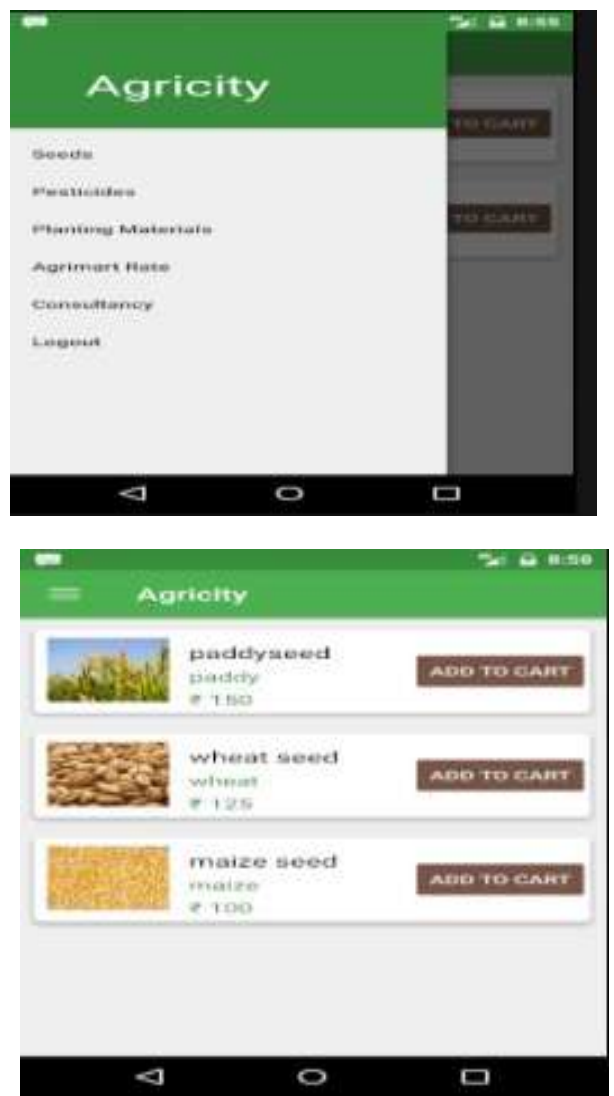

Fig 3: Seeds pages.
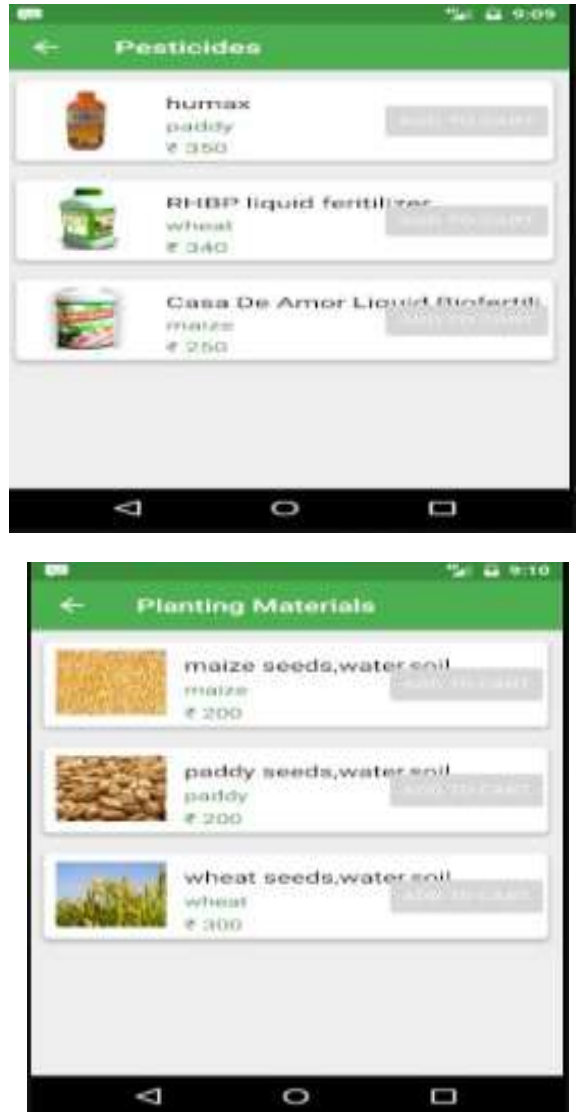

Fig 4: Pesticides \& Planting Materials 

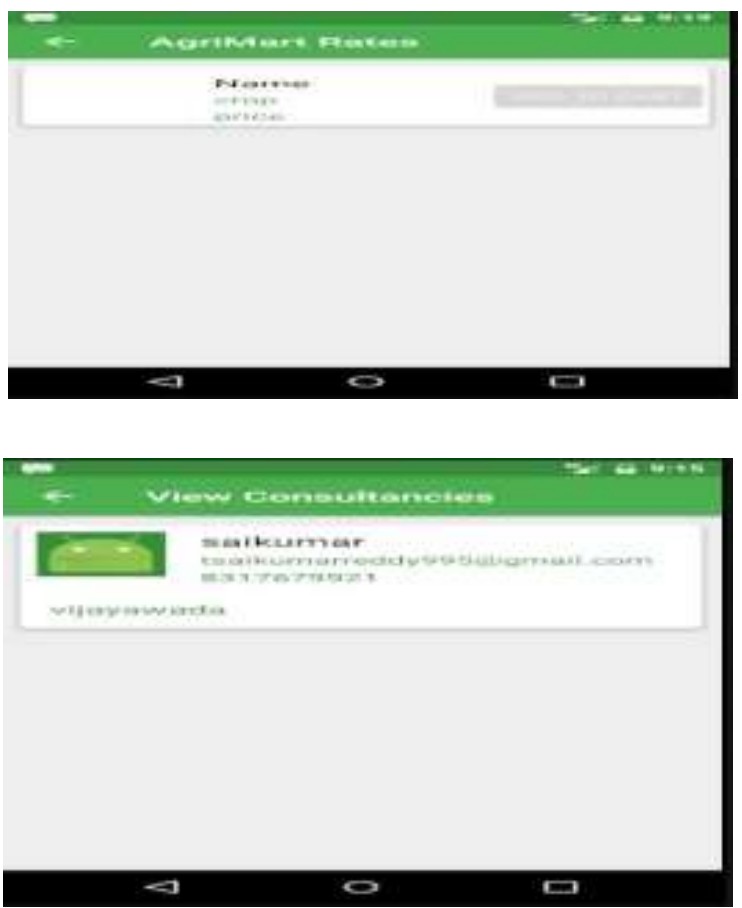

Fig 5: Agrimart Rate \& Consultancies

\section{CONCLUSION}

The main aim of our agricultural information system is to establish communication between farmers and agricultural experts and agricultural product marketing by information technology. The information is based on farmers needs, internet used as a mode to transfer the advanced agricultural information to the farming community. Farmers can be illiterate and speak a local language to our consultancy and receive the required information. Lack of knowledge of agricultural information may weaken the support for public information funding as a major priority in agriculture. An increase in funding for public information should allow for an increase in the accessibility of public information to farmers. For effective dissemination of agricultural information in rural communities by extension staffs research institutes and other responsible persons.

\section{REFERENCES}

[1]. Peter Namisiko and Moses Aballo "Current Status of e-Agriculture and Global Trends:A Survey Conducted inTransNzoia County, Kenya" in International Journal of Science and Research Volume 2 Issue 7, 2013

[2]. Marcel Fafchamps and Bart Minten "Impact of SMS-Based Agricultural Information on Indian Farmers" in Oxford journals VOL. 26, NO. 3, pp. 383-414, 2012

[3]. http://www.academia.edu/2980504/Agricultur al_information_systems_and_their_applicatio ns_for_development_of_agriculture_and_rura 1_community_a_review_study 